\title{
PLOS Neglected Tropical Diseases
}

\section{Access to morbidity management and disability prevention services for lymphatic filariasis in Luangwa district, Zambia: A mixed methods study. \\ --Manuscript Draft--}

\begin{tabular}{|c|c|}
\hline Manuscript Number: & PNTD-D-20-00131 \\
\hline Full Title: & $\begin{array}{l}\text { Access to morbidity management and disability prevention services for lymphatic } \\
\text { filariasis in Luangwa district, Zambia: A mixed methods study. }\end{array}$ \\
\hline Short Title: & Morbidity management \& disability prevention services for lymphatic filariasis. \\
\hline Article Type: & Research Article \\
\hline Keywords: & $\begin{array}{l}\text { Implementation Research; Lymphatic Filariasis; Morbidity management and disability } \\
\text { prevention }\end{array}$ \\
\hline Abstract: & $\begin{array}{l}\text { Introduction } \\
\text { Morbidity management and disability prevention (MMDP) services are essential for the } \\
\text { management of lymphoedema, elephantiasis and hydrocele that result from lymphatic } \\
\text { filariasis (LF) infection. However, there is little information on the access and utilization } \\
\text { of MMDP services in Zambia. This study sought to evaluate in status of LF morbidity, } \\
\text { utilization and factors affecting access to MMDP services in Luangwa District, Zambia. } \\
\text { Methods } \\
\text { A concurrent mixed methods study that included a descriptive cross-sectional survey of } \\
237 \text { patients, } 4 \text { Focus group discussions and } 20 \text { interviews were conducted. Data that } \\
\text { was collected included knowledge of LF morbidity, existing morbidity management } \\
\text { practices, health seeking behavior, factors affecting access to MMDP services and } \\
\text { potential areas of integration of MMDP services into the primary health care system. } \\
\text { Findings } \\
\text { The most common LF morbidity in the district was hydrocele. There were low levels of } \\
\text { knowledge on the causes, symptoms and disease management practices among the } \\
\text { different stakeholders. It was common for patients to seek care from both health } \\
\text { facilities and traditional healers. } 51.0 \% \text { of hydrocele patients had sought care from a } \\
\text { medical facility and } 62.5 \% \text { had gone traditional healers and utilization of surgeries for } \\
\text { hydrocele was poor. The main challenges to access included, limited understanding of } \\
\text { the disease, lack of awareness of existing MMDP services, unfavorable cultural and } \\
\text { social norms, poor motivation to maintain disease management practices and } \\
\text { inadequate resources at health facilities. Opportunities for integration included } \\
\text { embedding aspects of MMDP services in community structures, existing health } \\
\text { services and building capacity both health providers and community health workers. } \\
\text { Conclusion } \\
\text { Improving access to morbidity management and disability prevention services for LF } \\
\text { will require national programmes to effectively map patients and services within health } \\
\text { systems. This will streamline existing services and create the much-needed demand } \\
\text { for vulnerable and disadvantaged populations. }\end{array}$ \\
\hline \multicolumn{2}{|l|}{ Additional Information: } \\
\hline Question & Response \\
\hline $\begin{array}{l}\text { Financial Disclosure } \\
\text { Enter a financial disclosure statement that } \\
\text { describes the sources of funding for the } \\
\text { work included in this submission. Review } \\
\text { the submission guidelines for detailed } \\
\text { requirements. View published research } \\
\text { articles from PLOS NTDs for specific } \\
\text { examples. }\end{array}$ & $\begin{array}{l}\text { This study was funded by the Coalition for Operational Research on Neglected } \\
\text { Tropical Diseases (COR-NTD),(https://www.ntdsupport.org/cor-ntd/ntd- } \\
\text { connector/term/ntdsc), under Grant Number:NTD-SC \#163D and was awarded to CM. } \\
\text { The funders had no role in study design, data collection and analysis, decision to } \\
\text { publish, or preparation of the manuscript. }\end{array}$ \\
\hline This statement is required for submission & \\
\hline
\end{tabular}


and will appear in the published article if the submission is accepted. Please make sure it is accurate.

\section{Unfunded studies}

Enter: The author(s) received no specific funding for this work.

\section{Funded studies}

Enter a statement with the following details:

- Initials of the authors who received each award

- Grant numbers awarded to each author

- The full name of each funder

- URL of each funder website

- Did the sponsors or funders play any role in the study design, data collection and analysis, decision to publish, or preparation of the manuscript?

- NO - Include this sentence at the end of your statement: The funders had no role in study design, data collection and analysis, decision to publish, or preparation of the manuscript.

- YES - Specify the role(s) played.

\section{* typeset}

\section{Competing Interests}

The authors have declared that no competing interests exist.

Use the instructions below to enter a competing interest statement for this submission. On behalf of all authors, disclose any competing interests that could be perceived to bias this work-acknowledging all financial support and any other relevant financial or nonfinancial competing interests.

This statement will appear in the published article if the submission is accepted. Please make sure it is 
accurate. View published research articles from PLOS NTDs for specific examples.

\section{NO authors have competing interests}

Enter: The authors have declared that no competing interests exist.

\section{Authors with competing interests}

Enter competing interest details beginning with this statement:

I have read the journal's policy and the authors of this manuscript have the following competing interests: [insert competing interests here]

* typeset

\section{Data Availability}

Yes - all data are fully available without restriction

Authors are required to make all data underlying the findings described fully available, without restriction, and from the time of publication. PLOS allows rare exceptions to address legal and ethical concerns. See the PLOS Data Policy and $F A Q$ for detailed information.

A Data Availability Statement describing where the data can be found is required at submission. Your answers to this question constitute the Data Availability Statement and will be published in the article, if accepted. 
Important: Stating 'data available on request from the author' is not sufficient. If your data are only available upon request, select 'No' for the first question and explain your exceptional situation in the text box.

Do the authors confirm that all data underlying the findings described in their manuscript are fully available without restriction?

Describe where the data may be found in full sentences. If you are copying our The data underlying the results presented in the study are available from Taskforce for Global Health and will be made available through https://www.ntdsupport.org/corsample text, replace any instances of XXX ntd/ntd-connector/term/ntdsc at the completion of all study activities. with the appropriate details.

- If the data are held or will be held in a public repository, include URLs, accession numbers or DOls. If this information will only be available after acceptance, indicate this by ticking the box below. For example: All $X X X$ files are available from the $X X X$ database (accession number(s) $X X X, X X X$.).

- If the data are all contained within the manuscript and/or Supporting Information files, enter the following: All relevant data are within the manuscript and its Supporting Information files.

- If neither of these applies but you are able to provide details of access elsewhere, with or without limitations, please do so. For example:

Data cannot be shared publicly because of [XXX]. Data are available from the $X X X$ Institutional Data Access / Ethics Committee (contact via $X X X$ ) for researchers who meet the criteria for access to confidential data.

The data underlying the results 
presented in the study are available

from (include the name of the third party and contact information or URL).

- This text is appropriate if the data are owned by a third party and authors do not have permission to share the data.

* typeset

Additional data availability information: 
1 Full title: Access to morbidity management and disability prevention services for lymphatic

2 filariasis in Luangwa district, Zambia: A mixed methods study.

3

4 Short title: Morbidity management $\&$ disability prevention services for lymphatic filariasis 5

6 Authors and affiliations

7 Patricia Maritim ${ }^{1 *}$, Adam Silumbwe ${ }^{2 \pi}$, Joseph Mumba Zulu ${ }^{1 \pi}$, George Sichone ${ }^{3 \pi}$, Charles

8 Michelo $^{6 \%}$

$9{ }^{1}$ Department of Health Promotion and Education, University of Zambia School of Public 10 Health, Lusaka, Zambia.

$11{ }^{2}$ Department of Health Policy and Management, University of Zambia School of Public Health, 12 Lusaka, Zambia.

$13{ }^{3}$ Participatory Research and Innovations Management, Lusaka, Zambia.

14

15 Corresponding Author

16 *Patricia Maritim: triciamarie20@gmail.com

17

18 "These authors contributed equally to this work.

19 \& These authors also contributed equally to this work

20

21 


\section{Abstract}

\section{Introduction}

24 Morbidity management and disability prevention (MMDP) services are essential for the

25 management of lymphoedema, elephantiasis and hydrocele that result from lymphatic filariasis

26 (LF) infection. However, there is little information on the access and utilization of MMDP

27 services in Zambia. This study sought to evaluate in status of LF morbidity, utilization and factors

28 affecting access to MMDP services in Luangwa District, Zambia.

\section{Methods}

30 A concurrent mixed methods study that included a descriptive cross-sectional survey of 237 31 patients, 4 Focus group discussions and 20 interviews were conducted. Data that was collected

32 included knowledge of LF morbidity, existing morbidity management practices, health seeking

33 behavior, factors affecting access to MMDP services and potential areas of integration of MMDP

34 services into the primary health care system.

35 Findings

36 The most common LF morbidity in the district was hydrocele. There were low levels of

37 knowledge on the causes, symptoms and disease management practices among the different

38 stakeholders. It was common for patients to seek care from both health facilities and traditional

39 healers. $51.0 \%$ of hydrocele patients had sought care from a medical facility and $62.5 \%$ had gone

40 traditional healers and utilization of surgeries for hydrocele was poor. The main challenges to

41 access included, limited understanding of the disease, lack of awareness of existing MMDP

42 services, unfavorable cultural and social norms, poor motivation to maintain disease

43 management practices and inadequate resources at health facilities. Opportunities for

44 integration included embedding aspects of MMDP services in community structures, existing

45 health services and building capacity both health providers and community health workers. 


\section{Conclusion}

47 Improving access to morbidity management and disability prevention services for LF will require

48 national programmes to effectively map patients and services within health systems. This will

49 streamline existing services and create the much-needed demand for vulnerable and

50 disadvantaged populations.

\section{Author Summary}

52 Lymphatic filariasis (LF) infection if untreated usually results in fluid accumulation in the limbs

53 or breasts (lymphoedema) or genitalia (hydrocele) that is painful and causes great discomfort.

54 Morbidity management and disability prevention (MMDP) strategies such as surgery for

55 hydrocele, treatment of acute attacks and management of lymphoedema are necessary for the

56 management of the advances stages of LF. However very few countries including Zambia have

57 adequate information on the availability of MMDP services and quality of care for LF patients.

58 This study explored the utilization of MMDP services and factors that influence access to

59 appropriate care in a highly endemic region, Luangwa District, Zambia between February and

60 April 2019. Utilization of MMDP services was low. Factors affecting access to MMDP services

61 included lack of awareness of existing services, misconceptions about the disease, social and

62 cultural norms, gender, availability of resources and adherence to disease management practices.

63 Potential avenues for the integration of MMDP services into the existing health system include

64 included embedding aspects of MMDP services in community structures, existing health services

65 and building capacity both health providers and community health workers.

66

67

68

69 


\section{$71 \quad$ Background}

72 Lymphatic filariasis (LF), a neglected tropical disease, causes permanent disability through

73 chronic manifestations of lymphoedema and hydrocele accounting for 1.36 million disability

74 adjusted life years [1]. Globally more than 890 million people living in 49 endemic countries face

75 the risk of infection and an estimated 40 million have chronic manifestations of the disease [2].

76 Diseases control programmes have mostly targeted the interruption of LF transmission through

77 mass drug administration (MDA) whilst placing less emphasis on promoting morbidity management and disability prevention services (MMDP) for those presenting with chronic manifestations. The basic care package for MMDP services includes individual treatment to destroy microfilaria, treatment for episodes of adenolymphangitis (acute attacks), management

81 of lymphoedema to prevent disease progression and surgery for hydrocele [3]. Since 2000, MDA

82 for LF programs have delivered 7.7 billion cumulative number of treatments to people living in

83 endemic areas [2], whilst fewer lymphoedema and hydrocele patients have accessed to MMDP

84 services in the same period.

85 According to the World Health Organization (WHO), MMDP services should be included in 86 the basic primary healthcare package, and constitute an indicator for equity in progress towards

87 SDG 3.8 and universal health coverage. However, progress towards establishing and streamlining MMDP services still remains considerably slow, particularly in the Africa, which accounts for a considerable proportion of the LF burden. Out of the 34 LF endemic countries in the African

90 region, only 12 have indicated as having MMDP services for LF patients. WHO reports that even

91 data on MMDP services is rarely captured across countries. Global efforts to eliminate LF

92 through MDA are likely to stop beyond 2020, and strategic direction of diseases control will most likely pivot towards MMDP services. 
94 In Zambia, LF is a public health concern as 87 of 118 districts are considered endemic [2, 4]. MDA for LF was first piloted in Western Province in 2014, and then scaled up nationally in

962015 with annual campaigns to be implemented until 2019. In 2018, national MDA for LF coverage was reported at $90.8 \%$ [2]. Morbidity mapping of LF patients has been concurrently conducted with the implementation of MDA for LF campaigns. The mapping exercise has shown that there are many cases of hydrocele and lymphedema spread across all ten provinces in the country. However, there is limited information of what MMDP services are available, where and

101 how LF patients are accessing them.

102 The Elimination of Neglected Tropical Diseases National Masterplan 2019-2023 has placed huge 103 emphasis on disability prevention due to LF. However, Zambia currently lacks a comprehensive 104 national MMDP strategy for LF or suitable indicators to monitor the provision of MMDP services 105 [4]. As part of efforts to generate evidence to guide MMDP strategy formulation, the Ministry of 106 Health in collaboration with the University of Zambia, School of Public Health developed a pilot 107 initiative using implementation research approaches to identify the most appropriate 108 mechanisms through which MMDP services can be streamlined and integrated into existing 109 primary health care systems in Luangwa District to inform potential scale up.

110 Implementation research plays a vital role in Neglected tropical disease control programmes as 111 it makes it possible to identify optimum conditions that promote implementation success. By 112 exploring the process through which disease control interventions are implemented and the ways 113 in which contextual factors may affect implementation. The pilot initiative used an embedded 114 implementation research approach whereby a range of stakeholders including programme 115 implementers, researchers and communities living in the endemic district were involved in all 116 phases of the pilot initiative from problem identification, mapping of the status of MMDP 117 services, validation of findings, intervention development and implementation. 
118 This study presents the findings of a preliminary baseline assessment conducted prior to the

119 development and implementation of the pilot initiative. Specifically, this paper reports on the

120 perspectives of health providers, community members and patients on their knowledge of LF

121 morbidity, morbidity management practices, health seeking behavior, factors affecting access to

122 MMDP services and potential areas of integration of MMDP services into the existing primary

123 health systems in Luangwa District. This information would be used to tailor the components

124 of the MMDP intervention such as the design training curriculums, health education and

125 community sensitization materials, strengthening the data management and referral structures

126 as well as identification of potential areas for integration.

\section{Methods}

\section{Study design}

129 This was a concurrent mixed method study conducted as a formative assesment for a larger study

130 that sought to develop and implement an integrated health system intervention to improve access

131 of MMDP services for LF patients within Luangwa District of Lusaka, Zambia.

\section{Study setting}

133 The study was conducted between February and April 2019 in Luangwa District, which has one

134 of the highest prevalence of the Circulating Filarial Antigen [5] as it found along the Luangwa 135 river valley. The district is predominantly rural with an estimated population of 31,665 , covered

136 by a total of 17 health facilities.

\section{Baseline mapping of patients}

138 Prior to conducting the study, a census of LF was done to map the distribution of cases across

139 the health facility catchment areas and formed the basis for sampling for the data collection.

140 Community health workers (CHWs) went from household to household identifying patients

141 who exhibited signs of lymphoedema, elephantiasis and hydrocele. 


\section{Patient descriptive cross-sectional survey}

143 A total of 237 patients were identified during the mapping exercise all of whom were invited to

144 take part in the study. The recruitment of study participants for the patient survey was done by 145 the CHWs, within the health facilities and surrounding communities with the help of the study

146 team and staff from the District Health Office. Trained CHWs administered a structured KAP

147 questionnaire. All patients who were identified during the mapping exercise agreed to take part

148 in the study. Interviews were held within the homes LF patients to ensure that their privacy and

149 confidentiality was upheld.

150 Focus group discussions

151 There were 4 focus group discussions (FGD) held in Luangwa Boma and Mphuka health facility

152 catchment areas that had the highest number of patients in the district. The FGDs were an 153 opportunity to collect information on community level understanding of the chronic stages of

154 LF, practices used to manage the conditions and access to health care.

155 Participants were conveniently sampled from the communities living in the catchment areas and included LF patients. Potential study participants were identified by community health workers

157 working in the catchment areas and were invited by telephone to participate by the health facility in-charges. In terms of composition, the FGDs were mixed with regard to age but were separated by gender ( 2 male and 2 female.) The gender separation was essential given that in the

160 communities living in the area, hydrocele is considered a sensitive topic of discussion and this

161 would encourage community members to speak freely. Each FGD had between 10 and 12

162 participants. The FGDs were conducted at the health facility but away from patient areas to

163 ensure the privacy of the participants and avoid interruptions. The FGDs lasted for 1 hour 30

164 minutes to 2 hours.

165 Interviews 
167 well as community health workers $(n=8)$, health facility staff $(n=8)$ and traditional leaders $(n=8)$

168 from 8 health facility catchment areas which had the highest number of LF patients in the district

169 - Luangwa Boma, Mpukha, Katondwe, Kanemela, Chitope, Kasinsa, Luangwa District Hospital

170 and Mandombe. Participants were purposively sampled based on their involvement in the

171 implementation of LF elimination programmes in the area. The interviews were used identify

172 health system factors related to access of MMDP services in the district. A total of twenty

173 interviews were done with participants invited to take part either through telephone calls or face

174 to face. Interviews were conducted at the respective health facilities and the Luangwa district

175 Health Offices. Interviews lasted between 45 minutes to an hour.

176 Data collection

177 The KAP questionnaire included questions on knowledge of lymphoedema such as symptoms,

178 causes and prevention measures, morbidity management practices, health seeking behaviour and

179 factors affecting their access to care. Factors affecting access to care in the questionnaire,

180 Interview and FGD guides were adapted from Levesque et al's conceptual framework on patient

181 centered access to health care [6]. Prior to data collection the data collection tools were pretested

182 to ensure their suitability and changes made where necessary. All FGDs and interviews were

183 audio recorded with the consent of the study participants. In addition, field notes were taken

184 during the course of the interviews. Data collection was done in English and Nyanja to ensure

185 that all participants were able to articulate their perspectives as comprehensively as possible.

\section{Data analysis}

187 All recordings were transcribed verbatim and those done in Nyanja were translated to English.

188 A framework analysis approach was applied to analyze the data [7] using Levesque et al's

189 conceptual framework on patient centered access to health care and has been used in health 
systems and policy research $[6,8,9]$. The framework defines access as the "opportunity to reach

191 and obtain appropriate health care services in situations of perceived need for care." It proposes

192 that from a health care provider perspective, access to care can be categorized into five

193 dimensions; approachability, acceptability, availability and accommodation, affordability and

194 appropriateness. Barriers and facilitators can impact these dimensions which in turn affects an

195 individual's ability to utilize health services. By affecting their ability to perceive, ability to seek,

196 ability to reach, ability to pay and ability to engage with the available health services [6]. These

197 demand and supply side dimensions formed the coding tree from which predetermined themes

198 and sub themes were extracted using both inductive and deductive methods to ensure that

199 existing and emergent themes were exhaustively identified. Preliminary coding of the transcripts

200 was done by PM and AS. JZ reviewed the coding lists to ensure that identified codes were aligned

201 to the to the framework. Subsequently PM, AS and JZ jointly reviewed the codes and developed

202 the final codebook.

203 Quantitative data from the survey was entered in excel template, cleaned to promote data

204 correctness and thereafter exported to STATA version 13 where it was analysed. Baseline

205 measures of the different knowledge, awareness and practice questions were summarised using

206 descriptive statistics. The data was reported as proportions and frequencies. The data the

207 qualitative and quantitative analysis was then integrated to provide a comprehensive picture of

208 MMDP in the district. Comparisons to other studies done in LF endemic countries looking at

209 MMDP, was done to examine the congruence of the identified factors with existing literature

210 and to gauge the strength of the analysis.

211 Validation of study findings

212 A stakeholder meeting was held to validate the findings of the study and to confirm that the

213 information that they provided was accurately reflected in the transcripts. Fifty participants took 
214 part in the workshop including Local Chiefs, traditional leaders, Ministry of health staff and

215 CHWs. Another outcome of the stakeholder validation meeting was the identification of

216 avenues for potential integration of MMDP services into the health system within the district.

217 Participants worked together to identify health system strengthening approaches through

218 identifying underlying mechanisms necessary for successful integration by detailing key health

219 messages, necessary actors, existing referrals pathways, reporting systems, monitoring and

220 evaluation systems and mentorship for health workers.

221 Ethics statement

222 Ethical approval was sought from University of Zambia Biomedical Research Ethics Committee

223 (REF.017-11-18) and the National Health Research Authority under the Ministry of Health,

224 Zambia. All participants were informed of the purpose of the assessment, details of study

225 procedures including freedom to withdraw, potential benefits and risks, prior to the

226 commencement of data collection. Thereafter written informed consent was obtained as all

227 participants were 18 years and above.

228 Results

229 Characteristics of patients.

230 There were 237 individuals sampled during the mapping exercise and the patient survey, and 231 from these, 199 (85.4\%) had hydrocele, 27 (11.6\%) had lymphedema and 7 (3.0\%) had both

232 lymphoedema and hydrocele. A majority of the participants were male (90.7\%). The mean age

233 was 46.9 (S.D 21.4). Most of the survey participants practiced Christianity (96.2\%), were married

$234(66.2 \%)$ and had lived in the area for more than 5 years $(86 \%$.) For most of the study participants

235 the highest level of education that they had attended was primary school (70.5\%) as shown in 236 Table 1.

237 Table 1. Demographic characteristics of LF Patients in Luangwa District 


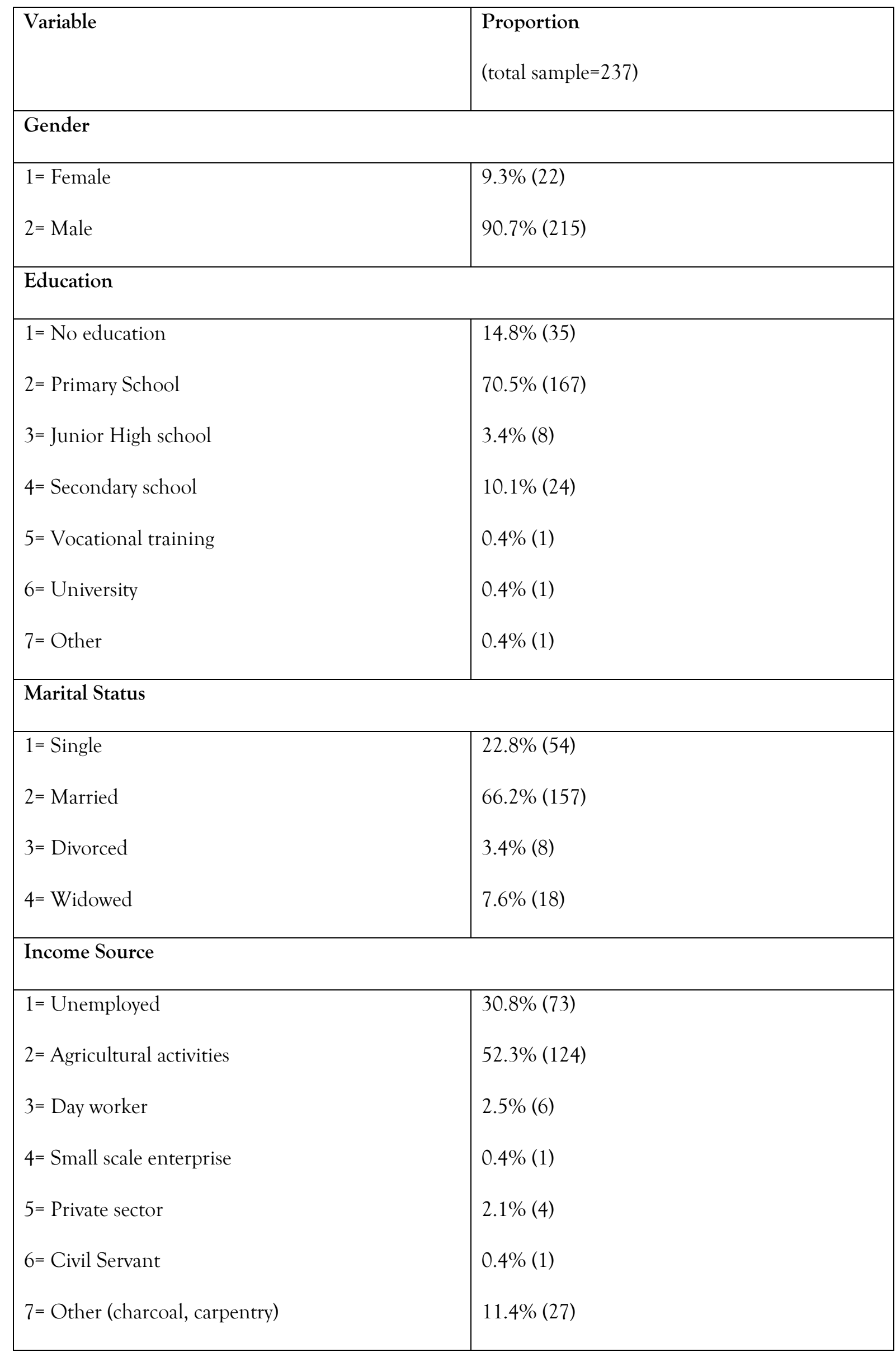




\begin{tabular}{|c|c|}
\hline \multicolumn{2}{|l|}{ Monthly Income } \\
\hline $1=<1000$ kwacha & $68.8 \%(161)$ \\
\hline $2=1000-4000$ kwacha & $4.7 \%(11)$ \\
\hline $3=4000-10000$ kwacha & $0.4 \%(1)$ \\
\hline $4=>10000$ kwacha & $3.8 \%(9)$ \\
\hline $5=I$ don't know & $22.2 \%(52)$ \\
\hline \multicolumn{2}{|c|}{ Duration they have lived in study area } \\
\hline$<1$ year & $4.2 \%(10)$ \\
\hline $1-5$ years & $8.4 \%(20)$ \\
\hline$>5$ years & $86 \%(203)$ \\
\hline $5^{*}$ & $1.3 \%(3)$ \\
\hline \multicolumn{2}{|l|}{ Available transport } \\
\hline $1=\mathrm{Car}$ & $3(1.3)$ \\
\hline $2=$ Bicycle & $46(19.8)$ \\
\hline $3=$ Motorbike & $1(0.4)$ \\
\hline $4=$ Walking & $176(75.9)$ \\
\hline $5=$ Other & $6(2.6)$ \\
\hline \multicolumn{2}{|c|}{ Transport to the health facility } \\
\hline $1=\mathrm{Car}$ & $2(0.9)$ \\
\hline $2=$ Bicycle & $12(5.4)$ \\
\hline $3=$ Motorbike & $19(8.6)$ \\
\hline $4=$ Walking & $62(28.1)$ \\
\hline $5=$ Other & $126(57.0)$ \\
\hline
\end{tabular}


240 The communities have local names for hydrocele, which is referred to as tumbu. There were

241 various cultural beliefs surrounding the causes of the chronic stages of LF. During the FGDs,

242 some of the common causes of lymphoedema and elephantiasis that were described included the

243 belief that the conditions were hereditary and was passed through family lines, eating food that

244 had not been warmed properly, contact with animal faeces, using traditional medicine such as

245 local herbal aphrodisiacs mutoto and children vomiting on their mothers during breastfeeding.

246 Reported causes of hydrocele were largely similar to those given for lymphoedema but also

247 included having sexual contact with women who were menstruating or who've had a miscarriage,

248 being bewitched, men using pounding sticks after they have been used by women, sitting on

249 chairs which patients have used and sitting on stones that women use during food preparation.

250 ".... Mostly what brings this disease is the careless sitting on things. For example, sitting on

251 the stones that women use for fire and also sitting on the stone that women use to grind or crush mealie meal......" [FGD1_Community Members].

253 Some of the FGD participants were able to correctly identify mosquitoes, which are common in

254 the area because of the presence of River Luangwa, as the disease-causing vectors. This could be due to community sensitisation exercises done as part of mass drug distribution rounds conducted between 2015 and 2018. However only 11.4\% patients could correctly identify mosquitoes as the vector that spreads the disease. When asked to rate their general understanding of their conditions, a majority of the patients stated that they had a poor understanding; ( $86.7 \%$ of lymphoedema and $77 \%$ of hydrocele patients). Most of the patients

260 and participants of the FGDs reported knowing patients who lived in their villages or were

261 members of their families and had lymphedema, elephantiasis or hydrocele.

262 Knowledge levels among traditional leaders, community-based volunteers and health care providers interviewed differed greatly. Generally, those who had been heavily involved in LF 
264 disease control activities such as MDA rounds were more likely to be knowledgeable about the

265 causes and symptoms of the disease, because they had received training and information to guide

266 implementation. Most of the traditional leaders interviewed reiterated the prevailing community

267 beliefs reported during the FGDs. For instance, during the stakeholder validation meeting, a

268 chief who was in attendance sought clarification on the means through which hydrocele is

269 transmitted and whether it be cured.

270 "....... Here we believe that if you are passing where there are faeces of animals especially this

271 rain season whether bush animals or village animals and it can be goats, cattle, sheep, pigs,

272 elephants or other animals the disease goes inside the nails making your legs swollen so even

273 if you treat hydrocele it can't be healed and that person will die at that old age with swollen

274 legs like that....” [KII5_ Traditional Leader].

275 Despite some healthcare providers having a basic understanding of the conditions and the

276 correct means of providing care, a considerable proportion of those who were interviewed and

277 who took part in the stakeholder validation meetings, acknowledged that they limited knowledge

278 of the disease and its management. Healthcare providers who came into regular contact with

279 patients coming to the facility or through community case identification exercises were better at

280 identifying signs and symptoms of lymphoedema, elephantiasis and hydrocele.

281 MMDP Practices and utilization of MMDP services

282 Health facility MMDP services. At the health facilities, available services are general in nature

283 without specific stand-alone activities for hydrocele and lymphedema. Not all health facilities in

284 the area have adequate resources to conduct hydrocelectomies and referrals are usually made to

285 either Katondwe Mission Hospital or Luangwa District Hospital. Severe cases are referred to the

286 University teaching Hospital in Lusaka. In the case of lymphoedema, the most readily available

287 services are pain relief and general health education with IEC materials on display in busy 
outpatient areas. The healthcare providers expressed that they also provided antibiotics to prevent secondary infection as a result of acute attacks as well as lymphatic draining to reduce

290 fluid density. Annual mass drug administration rounds were done to interrupt disease transmission. Despite surgical interventions being available at health facilities, utilisation was

292 low.

293 In terms of utilization of available services, 51,1\% of LF patients had sought care for their 294 condition at the health facility before. It was common to find that patients would go to the health

295 facility and to traditional healers. Community members pointed out that patients were more 296 likely to go to the traditional healers because they felt as though their remedies were a more 297 permanent solution compared to when they went to the facilities. Since once the medication 298 prescribed at the facility ran out their symptoms would reappear. One of the traditional healers 299 who took part in the validation meeting and had hydrocele himself, pointed out how common 300 it was for LF patients to seek remedies from him. Slightly more than half of the lymphoedema 301 patients (53.1\%) had previously sought medical help for their condition and $71.9 \%$ who had 302 gone to a traditional healer. Similarly, 51.0\% of hydrocele patients had sought care from a 303 medical facility and $62.5 \%$ had gone traditional healers. However, the tattoos and herbs

304 administered by the traditional healers would exacerbate the acute attacks forcing the patients to 305 go to the health facility.

306 The number of patients who had had surgery to reduce the swelling due to hydrocele was quite 307 low at $14.3 \%$. However, $58.6 \%$ of them were taking doxycycline as an alternative to surgery. Even 308 though the service is provided for free once they get a referral, the main reasons provided for 309 failure to have the surgeries done were insufficient money $(59.2 \%)$ and unaware of services $310 \quad(14.1 \%)$. 
311 Home based care strategies. The most common home-based strategies that health care facility

312 staff advised lymphoedema patients to do were exercise, elevation, improved hygiene owing to

313 the low economic situations that most of the patients came from, wearing baggy clothes, wound

314 management to prevent secondary infections and pain killers just to help the patients deal with

315 the immediate pain. Nevertheless, patients' knowledge of home-based care strategies that could

316 prevent their lymphoedema from worsening from the patient survey was found to be low. A

317 majority of lymphoedema patients (73.5\%) had never received any information on how to

318 manage their condition, despite more than half of them having been visited by a community-

319 based volunteer. With regards to availability of supplies to be able to effectively manage their

320 conditions from home, of the 22 patients with leg lymphedema only one had appropriate

321 footwear at home. Furthermore only $15.6 \%$ had bandages and $29 \%$ had either soap or water for

322 washing wounds. None of them had antibiotic ointments. Moreover $60 \%$ of the patients

323 admitted to not practicing any home-based care for their condition. Whereas those who did

324 mostly did exercises or used soap and water to clean affected areas. Community member

325 knowledge of home-based care strategies for lymphoedema and acute attacks was also found to

326 be low.

327 Factors affecting access to MMDP services

328 Approachability and ability to perceive need for care

329 Lack of awareness of existing services. A recurring theme across the focus group discussions and

330 the interviews is that most community members are not aware that there were MMDP services

331 available at the health facilities or that they could be able to take preventive measures through

332 home-based care strategies. Health talks provided by community health workers with the help of

333 LF patients as champions are being used as an opportunity to disseminate this information.

334 Resulting in patients who were initially hesitant about going to the health facility. Health 
335 education provided during outreach activities that were conducted as part of MDA were an

336 opportunity through which healthcare providers provided information on the diseases and more

337 community member were open to having their households sprayed during Indoor Residual

338 Spraying (IRS) exercises as part of prevention measures. Outside of MDA, community

339 sensitisation exercises tend to focus on diseases such as malaria, HIV/AIDS and maternal,

340 newborn and child related conditions as LF is not perceived to be a public health concern in the

341 district.

342 Belief about the disease. Due to the communal belief that LF is hereditary with some patients

343 having seen their parents and grandparents exhibiting symptoms and not seeking care, some

344 patients felt that it was unnecessary to seek care. Additionally, some patients were afraid to go

345 the health facility because they felt that their conditions had progressed to the more advanced

346 stages and that the services they would receive would not alleviate their symptoms

347 Misconceptions about the treatment process. In the case of hydrocele some thought that the

348 surgery provided would leave them in wheelchairs making it difficult for them to undertake their

349 regular duties.

350 Acceptability and ability to seek health care

351 Cultural beliefs. Hydrocele and lymphoedema are normalised in the district. Though LF patients

352 were considered to be in a pitiful state, FGD participants pointed out that it is common to see

353 hydrocele patients taking part in businesses such as fish mongering and farming.

354 Social norms. Having hydrocele is viewed as a marker of high social standing and men who had

355 it are more likely to be chosen as headmen as they are perceived to be old and wise. This has

356 acted as a deterrent to patients seeking care. A key deterrent to the uptake of surgical

357 interventions among hydrocele patients was the fear of loss of respect by their spouses. Some of

358 the patients thought that once the procedures had been done they would be incapable of having 
children which would make their wives not take them seriously. Some women in the communities whose husbands had hydrocele had left them due to lack of sexual satisfaction. “.... When they get sick they don't even go to the hospital because they are respected so much. Yes, they respect him a lot even if you reach somewhere he will be the first one to be given

367 Gender. According to the health care providers, it is more difficult to get men to come to the health facility, as compared to women and children despite hydrocele being the most common LF manifestation in the district. During the focus group discussions community members, shared the view that certain diseases, particularly to do with private parts are not meant to be shown to the opposite sex. As such hydrocele patients are embarrassed to access services and even if they do, they do not easily open up especially if they are being attended to by female health care providers. One of the suggestions given by community members on ways of increasing patients ability to seek care was having male healthcare providers handle hydrocele patients.

376 Stigmatisation. Fear of stigmatisation also inhibits patients' ability to seek care. During regular case identification exercises by healthcare providers, known hydrocele patients, who are approached for referral to the health facility would either deny having the condition or request to talk to them in private because they are afraid that they would be laughed at if other community members found out that they had hydrocele. The poor health seeking habits are compounded by low levels of education among communities living in the district. 
383 Geographic location. Luangwa District is very remote and a portion of the district is covered by

384 the Luangwa National Park. As a result, some communities in the district have to travel long

385 distances of up to 20 kilometers and more to the nearest health facility and their access may be 386 inhibited by wildlife attacks such as elephants from the Luangwa National Park. In addition,

387 roads to the health facilities are sometimes impassable and the most common means of transport

388 is bicycles which are inappropriate to transport lymphoedema and hydrocele patients. As such

389 some patients choose to stay home rather than go to the health facility.

390 Mobility. In addition, the district borders Mozambique and some patients are involved in

391 economic activities such as trade requiring them to travel. Therefore, when healthcare providers

392 conduct outreach and follow up visits at community level to provide some MMDP services they

393 do not find them at their homes.

394 Adequate resources. Procedures like hydrocelectomies require more qualified personnel like a

395 medical doctor, who were not common in remote areas where these patients are found. A

396 majority of the healthcare providers interviewed had not received any special training on how to

397 handle and manage patients with hydrocele and lymphedema, except for the normal training

398 provided during their formal training. They felt that they needed additional training to effectively

399 provide quality care to patients. In addition, medical supplies such as diagnostic tests necessary

400 for lymphedema and hydrocele management in most of the health facilities was inadequate.

401 Affordability and ability to pay for health care

402 Direct costs. Though patients are not expected to pay for the care that they receive at the health

403 facility, there is a perception among patients that they are still required to pay for interventions

404 such as the hydrocele surgery which dissuades them from seeking care.

405 Indirect costs. In the event that during assessment a patient's case is determined to be 406 complicated, they are referred to University Teaching Hospital in Lusaka. For most patients the 
associated costs needed for them to obtain the care they need including hiring transportation and out of pocket hospital expenses act as barriers to accessing care. The health facility catchment areas also cover neighboring villages in Mozambique and the residents in these villages usually save over time to be able to access care.

411 ".... If maybe the family doesn't have a bicycle and they have lymphoedema, some they have difficulties in walking so it is difficult for them to come and access the services if its not near and especially those from across the border they have to like save up money before they can come this side...." [IDI1_ Healthcare provider].

415 Opportunity costs. Moreover, most of those who live in the district are poor and when deciding

416 how best to prioritize available resources, they opt to use the minimal resources that are at their 417 disposal for food and basic necessities first before considering spending on health care. For most 418 of those involved in income generating activities such as fishing and trade, the opportunity cost 419 associated with taking time off to go to a health facility is not a preferred choice. According to 420 community members, the prevalent use of traditional healers and witchdoctors, often results in 421 sores and secondary infections that force patients to then go to the health facility which increases 422 the resources they spend on looking for effective treatment and management services.

\section{Appropriateness and ability to engage}

424 Adherence to MMDP practices. Progression of the symptoms of hydrocele and lymphoedema are dependent on proper long-term management and care. Patient motivation to maintain home

426 based care practices is critical to ensure that lymphoedema does not progress to elephantiasis and 427 that surgical interventions are undertaken early for hydrocele. A common sentiment expressed 428 during the FGDs and interviews was that many lymphoedema patients lose their motivation to 429 continue going to the facility for MMDP services which they felt was a waste of time, because 430 despite taking up medication over a long period their conditions stayed the same. They did not 
431 view the lack of progression of their symptoms to elephantiasis and fewer acute attacks as

432 favourable treatment outcomes as they wanted their symptoms fully cured.

433 Integration of MMDP services

434 Opportunities for integration.

435 Use of existing community structures. One of the main suggestions provided during the data

436 collection and the stakeholder validation meetings was use of community-based volunteers who

437 are rooted in the community and had been part of training for MDA, to deliver key messages

438 relating to MMDP. The inclusion of traditional leaders and chiefs in engaging community

439 members would help address some of the social and cultural beliefs that inhibited patients from

440 accessing care. Some traditional leaders would act as champions.

441 Training and capacity building. Provision of training to different health care providers and

442 community-based volunteers, was considered necessary in creating awareness of existing MMDP

443 services at the facilities and different home-based care strategies. Once trained they would be

444 better able to communicate the information to community members during outreach exercise,

445 health talks and when doing follow up visits with patients at their homes. As well as in schools

446 through the School health and Nutrition programme.

447 Integration into the existing healthcare services. Different suggestions were made by the

448 healthcare providers on which services would be most appropriate for integration. They felt that

449 lymphatic filariasis had some similarities with other diseases. Integrating into malaria programs

450 was proposed due to the similarities in the mode of transmission and ease of tailoring messages

451 around preventive and control measures. Water, Sanitation and Hygiene programmes were also

452 proposed due to the role of proper sanitation and hygiene in the prevention of secondary

453 infection.

454 DISCUSSION 
Lymphatic filariasis infection if untreated results, in advanced stages of lymphoedema,

456 elephantiasis and hydrocele which are not only debilitating but without care may result in

457 permanent disability. Morbidity management and disability prevention services provide an

458 opportunity through which patients could have access to management strategies that ensure their

459 conditions do not progress to more advanced stages. Allowing them to be fully involved in

460 community activities and undertake personal daily tasks.

461 In this study we explored perspectives towards MMDP practices within Luangwa district across

462 a diverse range of stakeholders from patients to service providers to identify the most appropriate

463 ways of providing care to LF patients within the existing health system in the district using

464 Levesque et al. framework [6]. As well as identify different determinants that may affect the

465 implementation on interventions that are aimed at providing MMDP services. We found that

466 knowledge of the causes of disease, sign/symptoms that mark the different diseases stages and

467 ways in which the disease can be managed through simple home-based care practices among

468 community members was very low. It was still common to find participants in the study who

469 thought that care practices would not have any effect on stopping disease progression. This is

470 despite ongoing health education efforts that are conducted in the area as part of mass drug

471 administration rounds. The negative effect of hydrocele on patients' marriages and sexual lives

472 is similar to what has been found in other settings [10].

473 Whilst the study was able to highlight the existence of certain healthcare services-like surgery-

474 that LF patients can access at the health facilities, it also demonstrated that access for the patients

475 still remains a huge challenge. The low level of priority given to morbidity management and

476 disability prevention services affects the ability of LF patients to seek appropriate care. Despite

477 the existence of various MMDP guidelines that guide health care providers and support

478 community and home-based care, it is common to find low knowledge levels within endemic 
479 districts. Community factors such as negative traditional beliefs around certain health services

480 like surgery play an important role with regards to shaping access and use of health services.

481 There is a need to provide training opportunities and strengthen referral systems to ensure

482 patients not only get appropriate care but that subsequent follow up is made possible.

483 Furthermore, MMDP programmes should include strategies that seek to empower LF patients

484 by ensuring that they get the required information, to access and use the services at the health

485 facilities.

486 Acknowledgements

487 We would like to acknowledge Edson Musonda and Frank Shamilimo from the Ministry of

488 Health who facilitated the study process. This work received financial support from the Coalition

489 for Operational Research on Neglected Tropical Diseases (COR-NTD), which is funded at The

490 Task Force for Global Health primarily by the Bill \& Melinda Gates Foundation, by the UK aid

491 from the British government, and by the United States Agency for International Development

492 through its Neglected Tropical Diseases Program.

493 


\section{References}

495 1. Kyu HH, Abate D, Abate KH, Abay SM, Abbafati C, Abbasi N, et al. Global, regional, 496 and national disability-adjusted life-years (DALYs) for 359 diseases and injuries and healthy life 497 expectancy (HALE) for 195 countries and territories, 1990-2017: a systematic analysis for the 498 Global Burden of Disease Study 2017. The Lancet. 2018;392(10159):1859-922.

499 2. World Health Organisation. Global programme to eliminate lymphatic filariasis: 500 progress report, 2018. . The Weekly Epidemiological Record 11th October 2019. 2019;2019, $50194: 457-72$.

502 3. World Health Organisation. Lymphatic filariasis: managing morbidity and preventing 503 disability: an aide-mémoire for national programme managers. 2013.

504 4. Ministry of Health,Zambia. Elimination of Neglected Tropical Diseases National 505 Masterplan 2019-2023. Lusaka, Zambia.: 2019.

506 5. Mwase ET, Stensgaard A-S, Nsakashalo-Senkwe M, Mubila L, Mwansa J, Songolo P, et 507 al. Mapping the geographical distribution of lymphatic filariasis in Zambia. PLoS neglected $508 \quad$ tropical diseases. 2014;8(2):e2714.

509 6. Levesque J-F, Harris MF, Russell G. Patient-centred access to health care: conceptualising 510 access at the interface of health systems and populations. International journal for equity in 511 health. 2013;12(1):18.

512 7. Ritchie J, Spencer L. Qualitative data analysis for applied policy research. Analyzing 513 qualitative data: Routledge; 2002. p. 187-208.

514 8. Hailemariam M, Fekadu A, Selamu M, Medhin G, Prince M, Hanlon C. Equitable access 515 to integrated primary mental healthcare for people with severe mental disorders in Ethiopia: a 516 formative study. International journal for equity in health. 2016;15(1):121. 
517 9. Davy C, Harfield S, McArthur A, Munn Z, Brown A. Access to primary health care 518 services for Indigenous peoples: A framework synthesis. International journal for equity in 519 health. 2016;15(1):163.

520 10. Babu BV, Mishra S, Nayak AN. Marriage, sex, and hydrocele: an ethnographic study on

521 the effect of filarial hydrocele on conjugal life and marriageability from Orissa, India. PLoS 522 neglected tropical diseases. 2009;3(4):e414. 